\title{
Measurement of the electron neutrino charged-current interaction rate on water with the T2K ND280 $\pi^{0}$ detector
}

K. Abe, ${ }^{1}$ J. Adam, ${ }^{2}$ H. Aihara,${ }^{3,4}$ C. Andreopoulos,${ }^{5,6}$ S. Aoki, ${ }^{7}$ A. Ariga,${ }^{8}$ S. Assylbekov, ${ }^{9}$ D. Autiero, ${ }^{10}$ M. Barbi, ${ }^{11}$ G. J. Barker, ${ }^{12}$ G. Barr, ${ }^{13}$ P. Bartet-Friburg,${ }^{14}$ M. Bass, ${ }^{9}$ M. Batkiewicz ${ }^{15}$ F. Bay ${ }^{16}$ V. Berardi, ${ }^{17}$ B. E. Berger,${ }^{9,4}$ S. Berkman, ${ }^{18}$ S. Bhadra, ${ }^{19}$ F. d. M. Blaszczyk, ${ }^{20}$ A. Blondel, ${ }^{21}$ S. Bolognesi, ${ }^{22}$ S. Bordoni, ${ }^{23}$ S. B. Boyd,${ }^{12}$ D. Brailsford ${ }^{24}$ A. Bravar, ${ }^{21}$ C. Bronner, ${ }^{4}$ N. Buchanan, ${ }^{9}$ R. G. Calland, ${ }^{4}$ J. Caravaca Rodríguez, ${ }^{23}$ S. L. Cartwright, ${ }^{25}$ R. Castillo, ${ }^{23}$ M. G. Catanesi, ${ }^{17}$ A. Cervera, ${ }^{26}$ D. Cherdack, ${ }^{9}$ N. Chikuma, ${ }^{3}$ G. Christodoulou, ${ }^{6}$ A. Clifton, ${ }^{9}$ J. Coleman, ${ }^{6}$ S. J. Coleman, ${ }^{27}$ G. Collazuol, ${ }^{28}$ K. Connolly, ${ }^{29}$ L. Cremonesi,${ }^{30}$ A. Dabrowska, ${ }^{15}$ R. Das, ${ }^{9}$ S. Davis, ${ }^{29}$ P. de Perio, ${ }^{31}$ G. De Rosa,${ }^{32}$ T. Dealtry, ${ }^{33,13}$ S. R. Dennis, ${ }^{12,5}$ C. Densham, ${ }^{5}$ D. Dewhurst, ${ }^{13}$ F. Di Lodovico, ${ }^{30}$ S. Di Luise, ${ }^{16}$ S. Dolan, ${ }^{13}$ O. Drapier, ${ }^{34}$ K. Duffy, ${ }^{13}$ J. Dumarchez,${ }^{14}$ S. Dytman,,${ }^{35}$ M. Dziewiecki,${ }^{36}$ S. Emery-Schrenk, ${ }^{22}$ A. Ereditato, ${ }^{8}$ L. Escudero,${ }^{26}$ T. Feusels,${ }^{18}$ A. J. Finch ${ }^{33}$ G. A. Fiorentini, ${ }^{19}$ M. Friend, ${ }^{37, *}$ Y. Fujii, ${ }^{37,}{ }^{*}$ Y. Fukuda, ${ }^{38}$ A. P. Furmanski, ${ }^{12}$ V. Galymov, ${ }^{10}$ A. Garcia, ${ }^{23}$ S. Giffin, ${ }^{11}$ C. Giganti, ${ }^{14}$ K. Gilje, ${ }^{2}$ D. Goeldi, ${ }^{8}$ T. Golan, ${ }^{39}$ M. Gonin, ${ }^{34}$ N. Grant,${ }^{33}$ D. Gudin, ${ }^{40}$ D. R. Hadley, ${ }^{12}$ L. Haegel,${ }^{21}$ A. Haesler, ${ }^{21}$ M. D. Haigh, ${ }^{12}$ P. Hamilton, ${ }^{24}$ D. Hansen, ${ }^{35}$ T. Hara, ${ }^{7}$ M. Hartz, ${ }^{4,41}$ T. Hasegawa, ${ }^{37, *}$ N. C. Hastings, ${ }^{11}$ T. Hayashino, ${ }^{42}$ Y. Hayato, ${ }^{1,4}$ R. L. Helmer, ${ }^{41}$ M. Hierholzer, ${ }^{8}$ J. Hignight, ${ }^{2}$ A. Hillairet, ${ }^{43}$ A. Himmel, ${ }^{44}$ T. Hiraki, ${ }^{42}$ S. Hirota ${ }^{42}$ J. Holeczek, ${ }^{45}$ S. Horikawa ${ }^{16}$ F. Hosomi ${ }^{3}$ K. Huang, ${ }^{42}$ A. K. Ichikawa, ${ }^{42}$ K. Ieki, ${ }^{42}$ M. Ieva ${ }^{23}$ M. Ikeda, ${ }^{1}$ J. Imber, ${ }^{34}$ J. Insler, ${ }^{46}$ T. J. Irvine, ${ }^{47}$ T. Ishida,${ }^{37, *}$ T. Ishii, ${ }^{37, *}$ E. Iwai, ${ }^{37}$ K. Iwamoto ${ }^{48} \mathrm{~K}$. Iyogi, ${ }^{1}$ A. Izmaylov, ${ }^{26,40}$ A. Jacob, ${ }^{13}$ B. Jamieson ${ }^{49}$ M. Jiang, ${ }^{42}$ S. Johnson, ${ }^{27}$ J. H. Jo, ${ }^{2}$ P. Jonsson, ${ }^{24}$ C. K. Jung, ${ }^{2, \dagger}$ M. Kabirnezhad ${ }^{50}$ A. C. Kaboth, ${ }^{24}$

T. Kajita, ${ }^{47, \dagger}$ H. Kakuno, ${ }^{51}$ J. Kameda, ${ }^{1}$ Y. Kanazawa, ${ }^{3}$ D. Karlen, ${ }^{43,41}$ I. Karpikov, ${ }^{40}$ T. Katori, ${ }^{30}$ E. Kearns, ${ }^{20, \dagger}$ M. Khabibullin, ${ }^{40}$ A. Khotjantsev, ${ }^{40}$ D. Kielczewska, ${ }^{52}$ T. Kikawa, ${ }^{42}$ A. Kilinski, ${ }^{50}$ J. Kim, ${ }^{18}$ S. King,${ }^{30}$ J. Kisiel, ${ }^{45}$

P. Kitching, ${ }^{59}$ T. Kobayashi, ${ }^{37, *}$ L. Koch, ${ }^{53}$ T. Koga, ${ }^{3}$ A. Kolaceke, ${ }^{11}$ A. Konaka, ${ }^{41}$ A. Kopylov, ${ }^{40}$ L. L. Kormos, ${ }^{33}$

A. Korzenev, ${ }^{21}$ Y. Koshio, ${ }^{54, \uparrow}$ W. Kropp,${ }^{55}$ H. Kubo, ${ }^{42}$ Y. Kudenko, ${ }^{40,}$ R. Kurjata, ${ }^{36}$ T. Kutter, ${ }^{46}$ J. Lagoda, ${ }^{50}$ I. Lamont, ${ }^{33}$ E. Larkin, ${ }^{12}$ M. Laveder, ${ }^{28}$ M. Lawe, ${ }^{33}$ M. Lazos, ${ }^{6}$ T. Lindner, ${ }^{41}$ C. Lister, ${ }^{12}$ R. P. Litchfield, ${ }^{12}$ A. Longhin, ${ }^{28}$ J. P. Lopez,${ }^{27}$ L. Ludovici, ${ }^{56}$ L. Magaletti, ${ }^{17}$ K. Mahn,${ }^{57}$ M. Malek,${ }^{25}$ S. Manly, ${ }^{48}$ A. D. Marino,${ }^{27}$ J. Marteau, ${ }^{10}$ J. F. Martin, ${ }^{31}$ P. Martins,${ }^{30}$

S. Martynenko, ${ }^{40}$ T. Maruyama, ${ }^{37, *}$ V. Matveev ${ }^{40}$ K. Mavrokoridis, ${ }^{6}$ E. Mazzucato, ${ }^{22}$ M. McCarthy, ${ }^{19}$ N. McCauley, ${ }^{6}$

K. S. McFarland ${ }^{48}$ C. McGrew, ${ }^{2}$ A. Mefodiev, ${ }^{40}$ C. Metelko, ${ }^{6}$ M. Mezzetto ${ }^{28}{ }^{28}$. Mijakowski, ${ }^{50}$ C. A. Miller, ${ }^{41}$

A. Minamino, ${ }^{42}$ O. Mineev, ${ }^{40}$ S. Mine, ${ }^{55}$ A. Missert, ${ }^{27}$ M. Miura, ${ }^{1,}$ S. Moriyama, ${ }^{1, \dagger}$ Th. A. Mueller, ${ }^{34}$ A. Murakami, ${ }^{42}$ M. Murdoch, ${ }^{6}$ S. Murphy, ${ }_{16}$ J. Myslik, ${ }^{43}$ T. Nakadaira, ${ }^{37, *}$ M. Nakahata, ${ }^{1,4}$ K. G. Nakamura, ${ }^{42}$ K. Nakamura, ${ }^{4, *}$

S. Nakayama, ${ }^{1, \dagger}$ T. Nakaya, ${ }^{42,4}$ K. Nakayoshi, ${ }^{37, *}$ C. Nantais, ${ }^{18}$ C. Nielsen,${ }^{18}$ M. Nirkko, ${ }^{8}$ K. Nishikawa, ${ }^{37, *}$ Y. Nishimura, ${ }^{47}$ J. Nowak, ${ }^{33}$ H. M. O'Keeffe, ${ }^{33}$ R. Ohta, ${ }^{37, *}$ K. Okumura, ${ }^{47,4}$ T. Okusawa, ${ }^{58}$ W. Oryszczak, ${ }^{52}$ S. M. Oser, ${ }^{18}$ T. Ovsyannikova, ${ }^{40}$ R. A. Owen, ${ }^{30}$ Y. Oyama, ${ }^{37, *}$ V. Palladino, ${ }^{32}$ J. L. Palomino, ${ }^{2}$ V. Paolone, ${ }^{35}$ D. Payne, ${ }^{6}$ O. Perevozchikov, ${ }^{46}$ J. D. Perkin, ${ }^{25}$ Y. Petrov, ${ }^{18}$ L. Pickard ${ }^{25}$ E. S. Pinzon Guerra, ${ }^{19}$ C. Pistillo, ${ }^{8}$ P. Plonski, ${ }^{36}$

E. Poplawska, ${ }^{30}$ B. Popov ${ }^{14,8}$ M. Posiadala-Zezula, ${ }^{52}$ J.-M. Poutissou, ${ }^{41}$ R. Poutissou, ${ }^{41}$ P. Przewlocki, ${ }^{50}$ B. Quilain, ${ }^{34}$ E. Radicioni, ${ }^{17}$ P. N. Ratoff, ${ }^{33}$ M. Ravonel, ${ }^{21}$ M. A. M. Rayner, ${ }^{21}$ A. Redij, ${ }^{8}$ M. Reeves, ${ }^{33}$ E. Reinherz-Aronis, ${ }^{9}$ C. Riccio, ${ }^{32}$ P. A. Rodrigues, ${ }^{48}$ P. Rojas, ${ }^{9}$ E. Rondio, ${ }^{50}$ S. Roth,${ }^{53}$ A. Rubbia, ${ }^{16}$ D. Ruterbories, ${ }^{9}$ A. Rychter, ${ }^{36}$ R. Sacco, ${ }^{30}$

K. Sakashita, ${ }^{37, *}$ F. Sánchez, ${ }^{23}$ F. Sato, ${ }^{37}$ E. Scantamburlo, ${ }^{21}$ K. Scholberg, ${ }^{44}$ S. Schoppmann, ${ }^{53}$ J. D. Schwehr, ${ }^{9}$ M. Scott, ${ }^{41}$ Y. Seiya ${ }^{58}$ T. Sekiguchi, ${ }^{37 * *}$ H. Sekiya, ${ }^{1, \dagger}$ D. Sgalaberna, ${ }^{16}$ R. Shah, ${ }^{5,13}$ A. Shaikhiev, ${ }^{40}$ F. Shaker, ${ }^{49}$ D. Shaw, ${ }^{33}$ M. Shiozawa, ${ }^{1,4}$ S. Short ${ }^{30}$ Y. Shustrov, ${ }^{40}$ P. Sinclair, ${ }^{24}$ B. Smith, ${ }^{24}$ M. Smy, ${ }^{55}$ J. T. Sobczyk ${ }^{39}$ H. Sobel, ${ }^{55,4}$ M. Sorel ${ }^{26}$ L. Southwell, ${ }^{33}$ P. Stamoulis, ${ }^{26}$ J. Steinmann, ${ }^{53}$ Y. Suda, ${ }^{3}$ A. Suzuki, K. Suzuki, ${ }^{42}$ S. Y. Suzuki, ${ }^{77,}{ }^{7}$ Y. Suzuki, ${ }^{4}$ R. Tacik, ${ }^{11,41}$ M. Tada, ${ }^{37, *}$ S. Takahashi, ${ }^{42}$ A. Takeda, ${ }^{1}{ }^{3}$. Takeuchi, ${ }^{7,4}$ H. K. Tanaka, ${ }^{1,}$ H. A. Tanaka, ${ }^{18, \|}$ M. M. Tanaka, ${ }^{37,{ }^{*}}$ D. Terhorst ${ }^{53}$ R. Terri, ${ }^{30}$ L. F. Thompson, ${ }^{25}$ A. Thorley, ${ }^{6}$ S. Tobayama, ${ }^{18}$ W. Toki, ${ }^{9}$ T. Tomura, ${ }^{1}$ C. Touramanis, ${ }^{6}$ T. Tsukamoto, ${ }^{37, *}$ M. Tzanov, ${ }^{46}$ Y. Uchida, ${ }^{24}$ A. Vacheret, ${ }^{13}$ M. Vagins, ${ }^{4,55}$ G. Vasseur, ${ }^{22}$ T. Wachala, ${ }^{15} \mathrm{~K}$. Wakamatsu, ${ }^{58} \mathrm{C}$. W. Walter, ${ }^{44}$ D. Wark, ${ }^{5,13}$ W. Warzycha, ${ }^{52}$ M. O. Wascko, ${ }^{24}$ A. Weber, ${ }^{5,13}$ R. Wendell, ${ }^{1, \dagger}$ R. J. Wilkes, ${ }^{29}$ M. J. Wilking, ${ }^{2}$ C. Wilkinson, ${ }^{25}$ Z. Williamson, ${ }^{13}$ J. R. Wilson, ${ }^{30}$ R. J. Wilson, ${ }^{9}$ T. Wongjirad,${ }^{44}$ Y. Yamada, ${ }^{37, *}$ K. Yamamoto, ${ }^{58}$ C. Yanagisawa, ${ }^{2,9}$ T. Yano, S. Yen, ${ }^{41}$ N. Yershov, ${ }^{40}$ M. Yokoyama, ${ }^{3,}$ J. Yoo, ${ }^{46}$ K. Yoshida, ${ }^{42}$ T. Yuan, ${ }^{27}$ M. Yu, ${ }^{19}$ A. Zalewska, ${ }^{15}$ J. Zalipska, ${ }^{50}$ L. Zambelli, ${ }^{37, *}$ K. Zaremba, ${ }^{36}$ M. Ziembicki, ${ }^{36}$ E. D. Zimmerman, ${ }^{27}$ M. Zito, ${ }^{22}$ and J. Żmuda ${ }^{39}$

(T2K Collaboration)

\footnotetext{
${ }^{1}$ University of Tokyo, Institute for Cosmic Ray Research, Kamioka Observatory, Kamioka, Japan

${ }^{2}$ State University of New York at Stony Brook, Department of Physics and Astronomy, Stony Brook, New York 11794, USA

${ }^{3}$ University of Tokyo, Department of Physics, Tokyo, Japan

${ }^{4}$ Kavli Institute for the Physics and Mathematics of the Universe (WPI), Todai Institutes for Advanced Study, University of Tokyo, Kashiwa, Chiba, Japan
} 
${ }^{5}$ STFC, Rutherford Appleton Laboratory, Harwell Oxford, and Daresbury Laboratory, Warrington, United Kingdom

${ }^{6}$ University of Liverpool, Department of Physics, Liverpool, United Kingdom ${ }^{7}$ Kobe University, Kobe, Japan

${ }^{8}$ University of Bern, Albert Einstein Center for Fundamental Physics, Laboratory for High Energy Physics (LHEP), Bern, Switzerland

${ }^{9}$ Colorado State University, Department of Physics, Fort Collins, Colorado 80523, USA

${ }^{10}$ Université de Lyon, Université Claude Bernard Lyon 1, IPN Lyon (IN2P3), Villeurbanne, France

${ }^{11}$ University of Regina, Department of Physics, Regina, Saskatchewan, Canada

${ }^{12}$ University of Warwick, Department of Physics, Coventry, United Kingdom

${ }^{13}$ Oxford University, Department of Physics, Oxford, United Kingdom

${ }^{14}$ UPMC, Université Paris Diderot, CNRS/IN2P3,

Laboratoire de Physique Nucléaire et de Hautes Energies (LPNHE), Paris, France

${ }^{15}$ H. Niewodniczanski Institute of Nuclear Physics PAN, Cracow, Poland

${ }^{16}$ ETH Zurich, Institute for Particle Physics, Zurich, Switzerland

${ }^{17}$ INFN Sezione di Bari and Università e Politecnico di Bari,

Dipartimento Interuniversitario di Fisica, Bari, Italy

${ }^{18}$ University of British Columbia, Department of Physics and Astronomy, Vancouver, British Columbia, Canada

${ }^{19}$ York University, Department of Physics and Astronomy, Toronto, Ontario, Canada

${ }^{20}$ Boston University, Department of Physics, Boston, Massachusetts 02215, USA

${ }^{21}$ University of Geneva, Section de Physique, DPNC, Geneva, Switzerland

${ }^{22}$ IRFU, CEA Saclay, Gif-sur-Yvette, France

${ }^{23}$ Institut de Fisica d'Altes Energies (IFAE), Bellaterra (Barcelona), Spain

${ }^{24}$ Imperial College London, Department of Physics, London, United Kingdom

${ }^{25}$ University of Sheffield, Department of Physics and Astronomy, Sheffield, United Kingdom

${ }^{26}$ IFIC (CSIC \& University of Valencia), Valencia, Spain

${ }^{27}$ University of Colorado at Boulder, Department of Physics, Boulder, Colorado 80309, USA

${ }^{28}$ INFN Sezione di Padova and Università di Padova, Dipartimento di Fisica, Padova, Italy

${ }^{29}$ University of Washington, Department of Physics, Seattle, Washington 98195, USA

${ }^{30}$ Queen Mary University of London, School of Physics and Astronomy, London, United Kingdom

${ }^{31}$ University of Toronto, Department of Physics, Toronto, Ontario, Canada

${ }^{32}$ INFN Sezione di Napoli and Università di Napoli, Dipartimento di Fisica, Napoli, Italy

${ }^{33}$ Lancaster University, Physics Department, Lancaster, United Kingdom

${ }^{34}$ Ecole Polytechnique, IN2P3-CNRS, Laboratoire Leprince-Ringuet, Palaiseau, France

${ }^{35}$ University of Pittsburgh, Department of Physics and Astronomy, Pittsburgh, Pennsylvania 15260, USA

${ }^{36}$ Warsaw University of Technology, Institute of Radioelectronics, Warsaw, Poland

${ }^{37}$ High Energy Accelerator Research Organization (KEK), Tsukuba, Ibaraki, Japan

${ }^{38}$ Miyagi University of Education, Department of Physics, Sendai, Japan

${ }^{39}$ Wroclaw University, Faculty of Physics and Astronomy, Wroclaw, Poland

${ }^{40}$ Institute for Nuclear Research of the Russian Academy of Sciences, Moscow, Russia

${ }^{41}$ TRIUMF, Vancouver, British Columbia, Canada

${ }^{42}$ Kyoto University, Department of Physics, Kyoto, Japan

${ }^{43}$ University of Victoria, Department of Physics and Astronomy, Victoria, British Columbia, Canada

${ }^{44}$ Duke University, Department of Physics, Durham, North Carolina 27708, USA

${ }^{45}$ University of Silesia, Institute of Physics, Katowice, Poland

${ }^{46}$ Louisiana State University, Department of Physics and Astronomy, Baton Rouge, Louisiana 70803, USA

${ }^{47}$ University of Tokyo, Institute for Cosmic Ray Research, Research Center for Cosmic Neutrinos, Kashiwa, Japan

${ }^{48}$ University of Rochester, Department of Physics and Astronomy, Rochester, New York 14627, USA

${ }^{49}$ University of Winnipeg, Department of Physics, Winnipeg, Manitoba, Canada

${ }^{50}$ National Centre for Nuclear Research, Warsaw, Poland

${ }^{51}$ Tokyo Metropolitan University, Department of Physics, Tokyo, Japan

${ }^{52}$ University of Warsaw, Faculty of Physics, Warsaw, Poland

${ }^{53}$ RWTH Aachen University, III. Physikalisches Institut, Aachen, Germany

${ }^{54}$ Okayama University, Department of Physics, Okayama, Japan

${ }^{55}$ University of California, Irvine, Department of Physics and Astronomy, Irvine, California 92697, USA

${ }^{56}$ INFN Sezione di Roma and Università di Roma "La Sapienza," Roma, Italy 
${ }^{57}$ Michigan State University, Department of Physics and Astronomy, East Lansing, Michigan 48824, USA

${ }^{58}$ Osaka City University, Department of Physics, Osaka, Japan

${ }^{59}$ University of Alberta, Centre for Particle Physics, Department of Physics, Edmonton, Alberta, Canada (Received 30 March 2015; revised manuscript received 8 May 2015; published 19 June 2015)

This paper presents a measurement of the charged current interaction rate of the electron neutrino beam component of the beam above $1.5 \mathrm{GeV}$ using the large fiducial mass of the $\mathrm{T} 2 \mathrm{~K} \pi^{0}$ detector. The predominant portion of the $\nu_{e}$ flux $(\sim 85 \%)$ at these energies comes from kaon decays. The measured ratio of the observed beam interaction rate to the predicted rate in the detector with water targets filled is $0.89 \pm 0.08$ (stat) \pm 0.11 (sys), and with the water targets emptied is $0.90 \pm 0.09$ (stat) \pm 0.13 (sys) The ratio obtained for the interactions on water only from an event subtraction method is $0.87 \pm 0.33$ (stat) \pm 0.21 (sys). This is the first measurement of the interaction rate of electron neutrinos on water, which is particularly of interest to experiments with water Cherenkov detectors.

DOI: 10.1103/PhysRevD.91.112010

PACS numbers: $13.15 .+\mathrm{g}$

\section{INTRODUCTION}

This paper reports a measurement of the ratio of the charged current $\nu_{e}$ event rate relative to the simulation with NEUT [1] event generator, version 4.1.4.2, for neutrino energies above $1.5 \mathrm{GeV}$ in the $\mathrm{T} 2 \mathrm{~K}$ beam. The interaction rate of electron neutrinos on water has never been measured at the neutrinos energies above $1.5 \mathrm{GeV}$, or at any other energies. The mean reconstructed energy of the selected neutrinos in the analysis presented in this paper is $2.7 \mathrm{GeV}$. The $\nu_{e}$ cross section has been measured on a liquid freon target for energies between 1.5 and $8 \mathrm{GeV}$ by Gargamelle [2] and on ${ }^{12} \mathrm{C}$ for energies around $32 \mathrm{MeV}$ at LANSCE [3]. Also at lower energies, the antielectron neutrino interactions have been measured by experiments near nuclear reactors. A review of neutrino cross section measurements can be found in [4].

The T2K experiment [5] was built with the primary goals of precisely determining the oscillation parameter $\theta_{13}$ via electron neutrino appearance, and of the parameters $\theta_{23}$ and $\Delta m_{32}^{2}$ via muon neutrino disappearance. The predominantly $\nu_{\mu}$ beam for these measurements is produced at the Japan Proton Accelerator Research Complex (J-PARC) in Tokai. The neutrinos from this beam are observed at a near detector, ND280, which is located $280 \mathrm{~m}$ downstream

\footnotetext{
*Also at J-PARC, Tokai, Japan.

${ }^{\dagger}$ Affiliated member at Kavli IPMU (WPI), the University of Tokyo, Japan.

*Also at Moscow Institute of Physics and Technology and National Research Nuclear University “MEPhI,” Moscow, Russia.

${ }^{\S}$ Also at JINR, Dubna, Russia.

"Also at Institute of Particle Physics, Canada.

"Also at BMCC/CUNY, Science Department, New York, New York, USA.

Published by the American Physical Society under the terms of the Creative Commons Attribution 3.0 License. Further distribution of this work must maintain attribution to the author(s) and the published article's title, journal citation, and DOI.
}

from the production target, where the neutrinos are not expected to have been affected by oscillations. The T2K far detector, Super-Kamiokande (SK), then measures the muon and electron neutrinos (and antineutrinos) after they have undergone a near maximal oscillation.

The oscillation probability for $\nu_{\mu} \rightarrow \nu_{e}$ depends on the mixing parameter, $\theta_{13}$, and on subleading effects that depend on the charge parity $(\mathrm{CP})$-violating phase, $\delta_{\mathrm{CP}}$, and on the mass hierarchy [6]. T2K has already observed the appearance of $28 \nu_{e}$ candidate events at the far detector with a $7.3 \sigma$ significance over a background expectation of $4.92 \pm 0.55$ events for $\theta_{13}=0$ [7]. The largest irreducible background for the appearance measurement comes from the predicted 3.2 intrinsic $\nu_{e}$ beam events.

In $\mathrm{T} 2 \mathrm{~K}$ the $\nu_{e}$ are expected to represent about $1.2 \%$ of the total neutrino flux [8]. The T2K $\nu_{\mu}$ beam is produced by magnetic focusing of pions and kaons produced by the interaction of a proton beam with a graphite target. The unavoidable $\nu_{e}$ component comes from the decay of muons from pion decay, and from kaon decay. In any long-baseline neutrino experiment proposed to measure $\mathrm{CP}$ violation and precisely measure neutrino oscillation parameters, the $\nu_{e}$ component of the beam will be the main source of background [9-11].

The measurement of the beam $\nu_{e}$ charged current $\left(\mathrm{CC}_{e}\right)$ interactions on a plastic scintillator and water target using ND280 tracker, was reported in [12]. This paper reports a direct measurement of this component of the charged current (CC) neutrino interactions in the ND280 $\pi^{0}$ detector (PØD) [13], which is located just upstream of the tracker. In this selection, the majority of the electron neutrinos were produced in kaon decay, and have energies above $1.5 \mathrm{GeV}$. The PØD detector has water targets that can be filled or emptied. Data were taken both with the targets filled to create a water target (water configuration), and empty to leave just air in place of the water target (air configuration). With data in the two configurations a subtraction analysis obtained the interaction rate just on water. 
Similar to the subtraction analysis presented here, a ratio analysis has been conducted by the Minerva collaboration for 2-20 GeV $\nu_{\mu}$ on $\mathrm{C}, \mathrm{Fe}$, and $\mathrm{Pb}$ compared to $\mathrm{CH}$ [14]. A subtraction analysis of the Minerva data is presented in the thesis of Tice [15]. Apart from the Minerva measurements, this appears to be the only other use of the subtraction analysis to date in neutrino scattering experiments.

The $\nu_{e}$ and $\nu_{\mu}$ come from the same pion to muon to electron decay chain, and lepton universality allows the expected rate of $\nu_{e}$ to be constrained by measuring the much larger flux of $\nu_{\mu}$. Details concerning the T2K beam flux measurement, and further information on recent measurements of $\nu_{\mu}$ interactions in the near detectors, can be found in Ref. [16].

One of the systematic uncertainties in long-baseline neutrino oscillation measurements using water Cherenkov detectors comes from model uncertainties in the meson exchange current for $\mathrm{C}$ versus for $\mathrm{O}$. Having measurements of neutrino interaction rates on water is therefore important. For a recent review of $\nu_{\mu}$ cross section measurements on various nuclear targets refer to the PDG [17]. The only measurements of $\nu_{\mu}$ neutrino interactions on water were reported by the $\mathrm{K} 2 \mathrm{~K}$ experiment for quasielastic interactions [18], and for reactions resulting in pions in the final state [19-22].

The paper is organized as follows. In Sec. II the PØD detector, used to do the measurement, is described. The electron selection, and expected backgrounds are then described in Sec. III. The particle identification (PID) to select electrons from muons in the PØD is a key component of this measurement, and will be described further in the section on event selection. The water subtraction method is then described in IV. The detector, reconstruction, flux and cross section systematic uncertainties in the measurement are reviewed in Sec. V. Finally the results of the rate measurement are presented in Sec. VI and a summary is in Sec. VII.

\section{ND280 $\pi^{0}$ DETECTOR}

The T2K ND280 $\pi^{0}$ Detector (PØD) is a scintillator based tracking calorimeter optimized to measure neutral current $\pi^{0}$ in the momentum range that contributes to backgrounds for $\nu_{e}$ appearance [13]. Refer to Fig. 4 of [13] for a picture of the PØD detector. The PØD is composed of layers of plastic scintillator alternating with water targets and brass sheets or lead sheets. The PØD sits in front of a tracking detector made up of two fine grain scintillator modules which serve as active targets placed between three time projection chambers. Both the PØD and tracking detector are surrounded by electromagnetic calorimeters and are in a $0.2 \mathrm{~T}$ magnetic field.

The PØD is constructed using 40 scintillator modules, each module is constructed with two perpendicular arrays of triangular scintillating bars and is approximately $38 \mathrm{~mm}$ thick. The scintillator modules are arranged in three regions. The most upstream and downstream regions of the detector are composed of seven modules interleaved with $4.5 \mathrm{~mm}$ thick sheets of stainless steel-clad lead that function as 4.9 radiation length electromagnetic calorimeters to improve the containment of photons and electrons. The central region serves as a target containing water. It has 25 water target layers that are $28 \mathrm{~mm}$ thick sandwiched between 26 scintillator modules and $1.3 \mathrm{~mm}$ brass sheets, positioned in between water targets and scintillator layers. The target region has a fiducial mass of approximately $1900 \mathrm{~kg}$ of water and $3570 \mathrm{~kg}$ of other materials.

The energy resolution of the PØD can be estimated from Monte Carlo studies by calculating the difference between true and reconstructed energy for many events. The energy resolution for electrons, after the selections described in III, is $16 \%$.

\section{EVENT SELECTION}

\section{A. Overview}

In this analysis, all the data collected between January 2010 and May 2013 except for a very small fraction of run III data, due to the magnetic horn current decrease which caused a failure in good spill preselection, are used. The data are subdivided into different run periods and PØD configurations as shown in Table I. The simulated data used in this analysis corresponds to 10 times the protons on target (POT) of the data, and reproduces the various experimental conditions of the different data-taking periods.

Neutrino interactions in ND280 are simulated with the NEUT [1] event generator, version 5.1.4.2. The generator covers a range of neutrino energy from several tens of $\mathrm{MeV}$ to hundreds of $\mathrm{TeV}$ and simulates all the nuclear targets present in ND280. In the simulated data, neutrino interactions are generated outside and within the full ND280 volume including all active and inactive material, providing information to understand the signal and backgrounds from interactions outside the ND280 fiducial volume. The details of the simulation process are described in [23].

TABLE I. Summary of T2K runs and the number of protons on target (POT) used in the analysis.

\begin{tabular}{|c|c|c|c|}
\hline $\mathrm{T} 2 \mathrm{~K}$ run & PØD configuration & Beam power $(\mathrm{kW})$ & POT $\left(\times 10^{19}\right)$ \\
\hline Run I & Water & 50 & 2.96 \\
\hline Run II & Water & 120 & 6.96 \\
\hline Run II & Air & 120 & 3.59 \\
\hline Run III & Air & 178 & 13.5 \\
\hline Run IV & Water & 178 & 16.5 \\
\hline Run IV & Air & 178 & 17.8 \\
\hline \multirow[t]{2}{*}{ Total } & Water & & 26.4 \\
\hline & Air & & 34.9 \\
\hline
\end{tabular}


Simulation of products of the neutrino interactions in the PØD is done using a GEANT 4.9.4 simulation [24-27]. The standard GEANT physics list for electromagnetic interactions is used in the simulation.

The analysis uses two reconstructed objects, a track and a shower. Within the PØD reconstruction algorithm, hits in the PØD scintillator layer associated with a reconstructed track classified as an electromagnetic track (typically electrons or photons) are forwarded to the shower reconstruction stage. Hits associated with a track that are classified as a light track (typically muon) or a heavy track (typically proton) are not forwarded to the shower reconstruction stage and cannot be reconstructed as a shower.

The signal events for the analysis are the charged current $\nu_{e}$ interactions in the PØD. A cut-based event selection using known reconstruction characteristics was tuned to maximize the product of efficiency and purity. To avoid bias, the selection strategy was developed based on Monte Carlo (MC) samples. Event displays of a typical $\mathrm{CC} \nu_{e}$ candidate and a $\pi^{0}$ background event selected in the analysis are shown in Fig. 1.

\section{B. Selection cuts}

The event selection strategy focuses on identifying single high-energy electron shower events with a vertex in the PØD. As a preselection, the reconstructed shower in the PØD must be in time with the beam bunch time. The PØD reconstruction searches for both tracks and showers with two independent algorithms, and the highest energy track and the highest energy shower are used in the analysis. The reconstruction algorithm builds tracks and showers from hits, but as the shower reconstruction occurs after the track reconstruction the algorithm needs to make sure that the hits shower reconstruction uses are the same hits the track reconstruction uses, for each single event. Therefore $80 \%$ of the hits associated with the track and shower are required to be the same.

In addition, events are selected where the angle of the reconstructed shower with respect to the $\mathrm{z}$-axis, which is approximately the beam axis, is less than $45^{\circ}$. The scintillator bars of the PØD have a triangular profile with angles of approximately $45^{\circ}$. Particles with an angle of more than $45^{\circ}$ with respect to the beam axis would therefore hit more than two adjacent bars in a layer. The PØD reconstruction algorithm currently only handles up to two adjacent bar hits in a layer, causing reconstruction failures for higher angle tracks.

For this analysis, only events with a reconstructed neutrino energy of $1.5 \mathrm{GeV}$ or more are selected. Reconstructed neutrino energy is calculated from the reconstructed electron energy and the electron angle using the quasielastic approximation. In this energy region, the majority of the $\nu_{e}$ flux arises from kaon decays and the PØD shows good performance to distinguish electrons
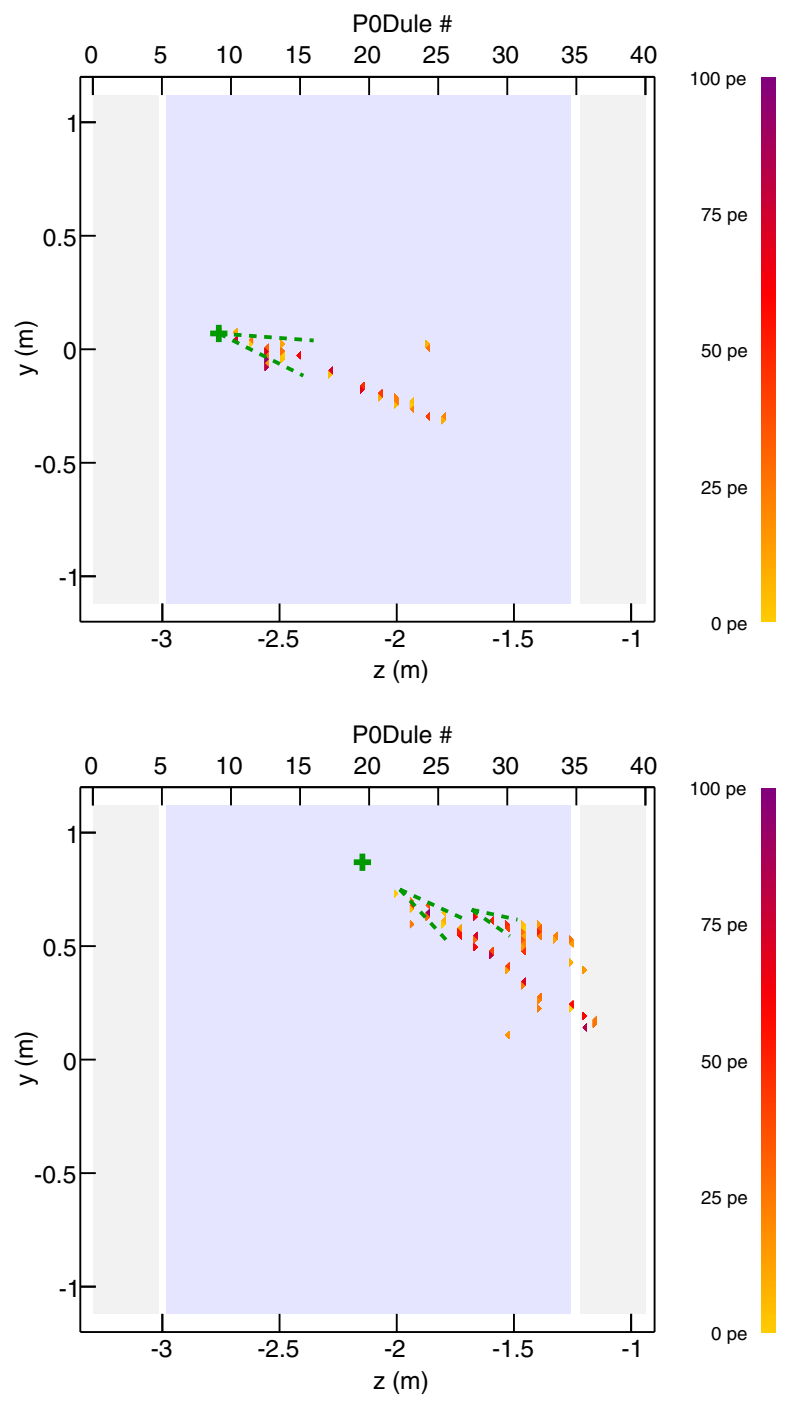

FIG. 1 (color online). Side view of a $\mathrm{CC} \nu_{e}$ event (top) and a $\pi^{0}$ background event (bottom) reconstructed in the PØD. Triangles are hits colored by the charge deposited, the green cross symbol shows the reconstructed shower vertex, and the green dashed lines show the cones of reconstructed showers.

from other particles. In addition, using a high neutrino energy cut improves the purity of the electron sample.

To reject muons, the median width of the selected track is used. In each scintillator layer, the energy-weighted standard deviation of the position of the hits reconstructed in the track is calculated as follows:

(1) If the two hits with the highest deposited energy are in adjacent strips, replace them with a single hit. The new hit's position is at the energy-weighted average position of the two original hits, and its energy is the sum of the energies of the original hits. Any other hits in the layers are left unchanged. This procedure gives layers with minimum ionizing tracks very small (almost always zero) width.

(2) The energy-weighted standard deviation of the hit positions is calculated for each layer. 
(3) Median width is the width of the middle layer after ordering by layer width.

The design of the PØD with layers of high density materials (brass and lead) causes electrons to shower. The reconstructed track of an electron is therefore typically wider than the reconstructed track of a muon. This feature can be used to distinguish muons and electrons with the median width of the reconstructed candidate track.

The track median width for events which pass all the selection criteria with the exception of the track median width cut is shown in Fig. 2 and indicates that most of the background muon events are rejected by this cut.

Similarly, to reject background events that contain neutral pions, a cut is applied to the median width of the selected shower. The shower reconstruction looks for hits in a cone from the reconstructed vertex position and combines them in one or more showers. It can happen that hits from several particles are combined in one reconstructed shower, especially when they are almost overlapping. The $\mathrm{P} \emptyset \mathrm{D} \nu_{e}$ analysis looks for events with a single electron. Events with a very wide candidate shower are
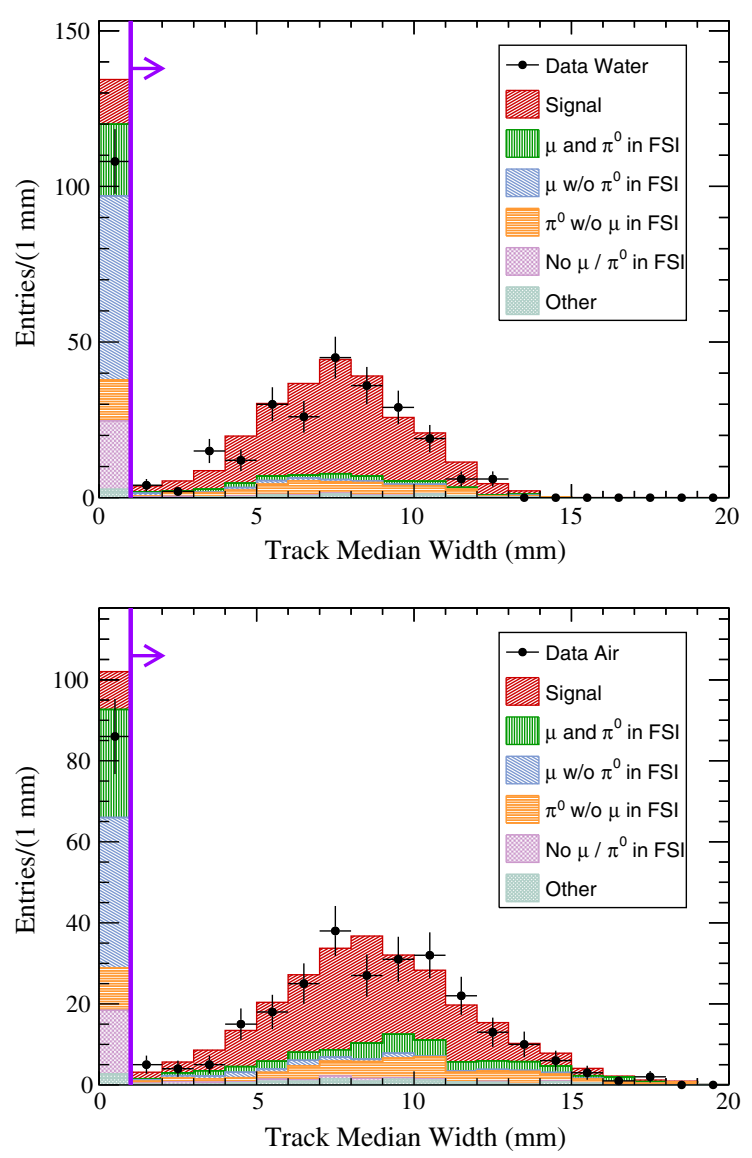

FIG. 2 (color online). Distribution of events which pass all the selection criteria with the exception of the track median width cut, for water (top) and air configuration (bottom). The vertical line shows the cut value used $(1 \mathrm{~mm})$. A sudden drop of events above $11 \mathrm{~mm}$ is an effect of shower median width cut. rejected, because such events are more likely background events with several particles. The shower median width is calculated the same way as the track median width. Distributions of events which pass all the selection criteria with the exception of the shower median width cut is shown in Fig. 3. It shows many $\pi^{0}$ background events are rejected with this cut.

Finally, a cut is applied to the fraction of the event's charge that is contained in the selected shower. To select $\mathrm{CC} \nu_{e}$ events with a high purity, the fraction of the event's charge contained in the candidate shower of exactly 1.0 is required, which selects only events with a single shower and without muonlike tracks in the final state.

\section{Selected event samples}

The selected number of events passing all cuts predicted by the simulation, both when the PØD is configured to contain water and air, together with the number of selected data events are presented in Table II. The water configuration simulation events are separated into on-water and
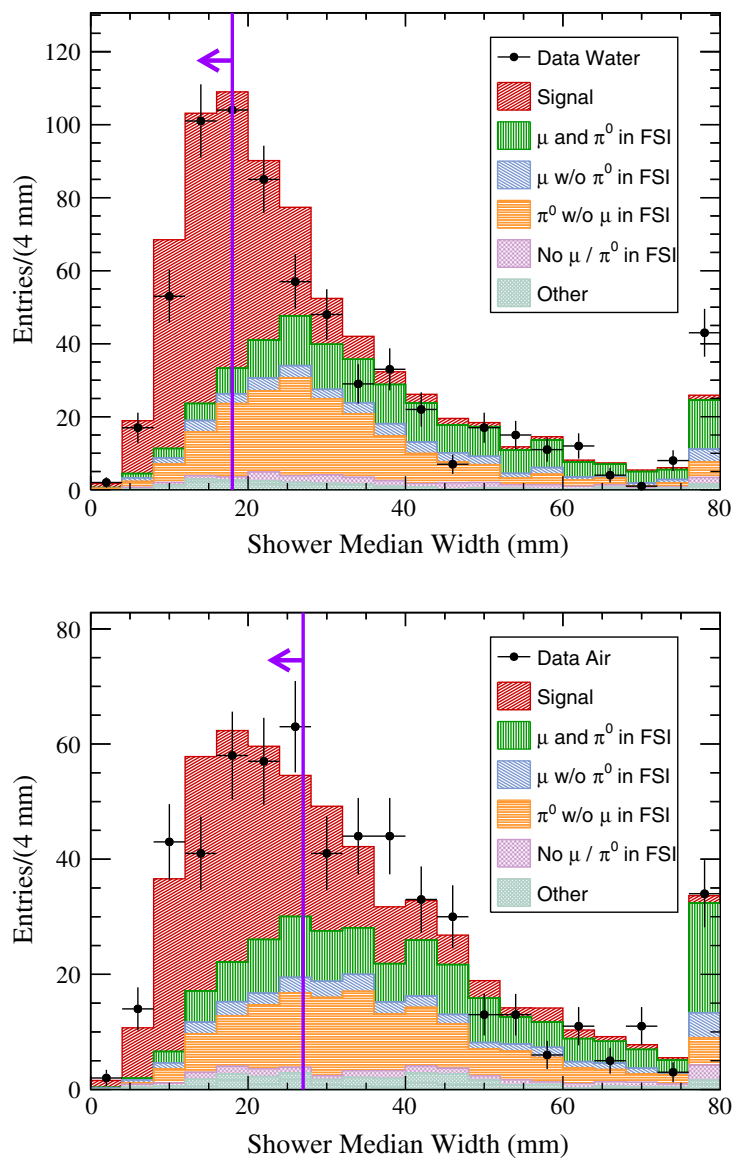

FIG. 3 (color online). Distribution of events which pass all the selection criteria with the exception of the shower median width cut, for water (top) and air configuration (bottom). The vertical line shows the applied cuts which are optimized for each configuration. 
TABLE II. The selected number of MC signal events, MC background events, and the total number of selected MC events normalized to data POT for water and air configuration are listed together with the selected data events. In addition, the water configuration MC events are split up in on-water and not-water events. The errors correspond to the statistical uncertainty due to the limited MC statistics.

\begin{tabular}{lrcrr}
\hline \hline & MC signal & MC background & MC total & Data \\
\hline Water & $196.1 \pm 4.8$ & $56.7 \pm 2.7$ & $252.8 \pm 5.5$ & 230 \\
On-water & $60.2 \pm 2.6$ & $14.5 \pm 1.3$ & $74.7 \pm 2.9$ & \\
Not-water & $135.9 \pm 4.0$ & $42.2 \pm 2.3$ & $178.2 \pm 4.6$ & \\
Air & $173.6 \pm 4.6$ & $97.4 \pm 3.6$ & $271.0 \pm 5.8$ & 257 \\
\hline \hline
\end{tabular}

TABLE III. The signal efficiencies $\epsilon$ and purities $p$ are listed for water and air configuration. Events of the PØD water configuration are split into events happening on-water and not-water. The errors correspond to the statistical uncertainty due to the limited MC statistics.

\begin{tabular}{lrc}
\hline \hline & \multicolumn{1}{c}{ Efficiency $\epsilon$} & \multicolumn{1}{c}{ Purity $p$} \\
\hline Water & $(10.9 \pm 0.3) \%$ & $(77.6 \pm 2.5) \%$ \\
On-water & $(9.8 \pm 0.4) \%$ & $(80.6 \pm 4.7) \%$ \\
Not-water & $(11.5 \pm 0.4) \%$ & $(76.3 \pm 3.0) \%$ \\
Air & $(11.0 \pm 0.3) \%$ & $(64.1 \pm 2.2) \%$ \\
\hline \hline
\end{tabular}

not-water events. On-water events are defined as events with true interaction vertex in the water, and not-water events have the true interaction vertex on scintillator, lead, brass, or other materials besides water. All events in the air configuration $\mathrm{MC}$ are not-water events as the water targets are drained.

\section{Efficiency and purity}

The efficiency $\epsilon$ and purity $p$ of the simulated electron neutrino signal events, for water and air configurations, are

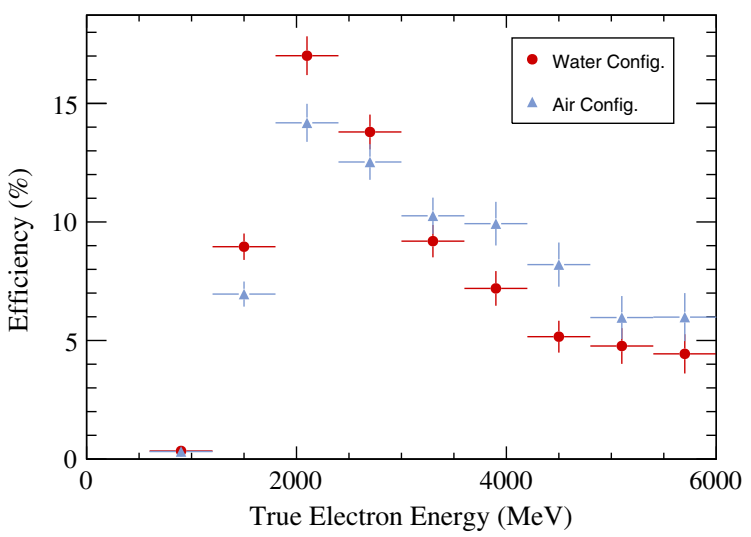

FIG. 4 (color online). Selection efficiency of signal events as a function of the true neutrino energy $E_{\text {true }}$ for water and air configuration. The error bars correspond to the uncertainties due to limited MC statistics. summarized in Table III. In the PØD water configuration, events are split into events happening on water (on-water) and events on scintillator, brass, and lead (not-water).

The selection efficiency of signal events as function of the true neutrino energy $E_{\text {true }}$ for PØD water and air configurations are shown in Fig. 4. The selection of low energy signal events is suppressed by the high neutrino energy cut at $1.5 \mathrm{GeV}$ while the selection of high energy signal events is suppressed by the shower median width cut and the shower charge fraction cut.

\section{WATER SUBTRACTION METHOD}

The measured $\nu_{e}$ interactions that were collected during PØD water and air configuration running are compared with the number of $\nu_{e}$ interactions predicted by the PØD water and air configuration MC, respectively. The measured number of $\nu_{e}$ interactions are extracted by subtracting the predicted MC background $B$ from the selected data events $D$, resulting in

$$
\begin{aligned}
N_{\mathrm{CC} \nu_{e}, \text { water }}^{\text {Data }} & =D_{\text {water }}-B_{\text {water }}, \quad \text { and } \\
N_{\mathrm{CC} \nu_{e} \text {,air }}^{\text {Data }} & =D_{\text {air }}-B_{\text {air }} .
\end{aligned}
$$

The background subtracted data are then divided by the predicted Monte Carlo signal $S$ to obtain the data/MC ratios for the water and air configurations:

$$
\begin{aligned}
R_{\text {water }} & =\frac{N_{\mathrm{CC} \nu_{e}, \text { water }}^{\text {Data }}}{S_{\text {water }}}, \text { and } \\
R_{\text {air }} & =\frac{N_{\mathrm{CC} \nu_{e}, \text { air }}^{\text {Data }}}{S_{\text {air }}^{\text {Dar }}} .
\end{aligned}
$$

To extract the measured number of on-water charged current $\nu_{e}$ interactions, the measured $\mathrm{CC} \nu_{e}$ interactions with PØD water and air configurations are compared by taking into account the different collected POT and the different reconstruction efficiencies for the water and the air data sample using

$$
\begin{aligned}
N_{\mathrm{CC}_{e}, \text { on-water }}^{\text {Data }}= & \left(D_{\text {water }}-B_{\text {water }}\right) \\
& -\frac{\epsilon_{\text {not-water }} \cdot \mathrm{POT}_{\text {water }}}{\epsilon_{\text {air }} \cdot \mathrm{POT}_{\text {air }}} \cdot\left(D_{\text {air }}-B_{\text {air }}\right) .
\end{aligned}
$$

In this formula, $\mathrm{POT}_{\text {water }}=2.64 \times 10^{20} \quad\left(\mathrm{POT}_{\text {air }}=\right.$ $3.49 \times 10^{20}$ ) is the collected data POT for the PØD water (air) configuration. The resulting data/MC ratio for onwater $\mathrm{CC} \nu_{e}$ interactions is given by

$$
R_{\text {on-water }}=\frac{N_{\mathrm{CC} \nu_{e}, \text { on-water }}^{\text {Data }}}{S_{\text {on-water }}}
$$




\section{SYSTEMATIC UNCERTAINTIES}

The systematic uncertainties in the measurements are divided into three categories: detector, reconstruction, and neutrino flux/cross section uncertainties. Control sample events to study systematic effects in the measurement have been studied, but often the events in these control samples are not used for the final systematic uncertainty evaluation. The control sample events were found to be too similar to the signal events, or did not have the same background as the signal events. For this reason a simple Kolmogorov Smirnov (KS) test is used for several of the systematic uncertainty tests, particularly where no deviation is indicated in the test.

\section{A. Detector systematic uncertainties}

The detector's as-built mass and its mass in the Monte Carlo are different. The masses for water and air configurations as well as different run periods also vary. These differences are incorporated in the analysis procedure by reweighting $\mathrm{MC}$ events with mass uncertainties estimated to be 0.01 for all configurations. Similarly, the fiducial volume and the alignment of the PØD is considered. Varying the fiducial volume by the MC vertex resolution and shifting in $\mathrm{PØD}$ alignment provides an estimate of the systematic uncertainties in data/MC ratios. The uncertainties obtained are smaller than 0.01 for all ratios making them negligible in this measurement.

Possible systematic effects on the reconstructed electron energy are also studied. The effects are investigated by changing the reconstructed energy scale to observe the differences in $\mathrm{CC} \nu_{e}$ data/MC ratios. The possible effects are as follows: 1. PØD material density and thickness, 2. drifts in the PØD response over time, and 3. the simulation (GEANT4) uncertainty in the electron energy deposition. It is assumed the water and air configuration are correlated for the PØD material density and thickness only. The resulting systematic uncertainties for water $\left(R_{\text {water }}\right)$, air $\left(R_{\text {air }}\right)$, and on-water $\left(R_{\text {on-water }}\right)$ are $0.05,0.05$, and 0.10 respectively.

\section{B. Reconstruction systematic uncertainties}

\section{Track PID}

As described earlier at the beginning of Sec. III, the classification of the reconstructed tracks is based on the PØD PID. Differences in the PID between data and MC can therefore cause systematic uncertainties in the $\mathrm{CC} \nu_{e}$ data/ $\mathrm{MC}$ ratios.

A PID study with stopping muons in the PØD was performed to estimate this uncertainty, and a map of misPID between a data sample and a simulation of stopping muons was constructed. To estimate the impact of the track PID uncertainty on the $\mathrm{CC} \nu_{e}$ data/MC ratios, the $\mathrm{MC}$ signal and background was weighted according to the uncertainty of the map. The systematic parameter values were randomly varied assuming that the water and air samples are uncorrelated and also that the signal and background uncertainties are uncorrelated. The uncertainties for water $\left(R_{\text {water }}\right)$, air $\left(R_{\text {air }}\right)$, and on-water $\left(R_{\text {on-water }}\right)$ were determined to be $0.05,0.05$, and 0.09 respectively.

\section{Track and shower median width}

To estimate the systematic uncertainty caused by the track median width, the plots with all selection criteria applied but failing the track median width cut (the $\mathrm{N}-1$ plots) are integrated, and a Kolmogorov-Smirnov test is performed to test if the data and the Monte Carlo event distributions are consistent [28,29]. The KolmogorovSmirnov test returns a p-value of $91.2 \%$ for water and $92.2 \%$ for air configuration indicating that there are no significant evidence for a shift between the data and MC event distributions. The systematic uncertainty due to the track median width cut is therefore negligible for this analysis.

The threshold of the shower median width cut is placed in a region with a large number of events. The systematic uncertainty on the measured shower median width therefore has a larger impact on the $\mathrm{CC} \nu_{e}$ data/ $\mathrm{MC}$ ratios than the track median width uncertainty does. To estimate the systematic uncertainty caused by the shower median width, the N-1 plots are integrated, and a Kolmogorov-Smirnov test is performed. The Kolmogorov-Smirnov test returns a p-value of $50.0 \%$ for water and $65.9 \%$ for air configuration. To determine a reasonable scaling factor range for Monte Carlo, different scaling factors from 0.9 to 1.1 were applied to Monte Carlo and the resulting p-values were studied. For a p-value of $68 \%$, the peak scaling factor ranged from 0.98 to 1.02 . The systematic effect on the $\mathrm{CC} \nu_{e}$ data/MC ratios for $R_{\text {water }}, R_{\text {air }}$, and $R_{\text {on-water }}$ coming from the shower median width are estimated by varying the scaling factor that is applied to the MC shower median width. The uncertainties obtained for $R_{\text {water }}, R_{\text {air }}$, and $R_{\text {on-water }}$ are $0.04,0.04$, and 0.08 respectively.

\section{Shower charge fraction}

To estimate the possible impact of systematic effects of the shower charge fraction on the analysis, additional reconstructed objects with low energy are studied. Such additional tracks or showers would cause an event to fail the shower charge fraction selection criteria. Looking at the event distribution of these events, the only hint for a systematic difference between data and MC appears in the highest bin of the air configuration. Events with a shower charge fraction between 0.98 and 1.00 which pass all other selection criteria are analyzed to estimate the systematic uncertainty. The data/MC difference in this region is considered to be the uncertainty on the $\mathrm{MC}$ events in the signal region, resulting in the systematic uncertainties for $R_{\text {water }}, R_{\text {air }}$, and $R_{\text {on-water }}$ of $0.01,0.04$, and 0.04 respectively. 
TABLE IV. Summary of systematic uncertainties on the $\mathrm{CC} \nu_{e}$ data/MC ratios for water $\left(R_{\text {water }}\right)$, air $\left(R_{\text {air }}\right)$, and on-water $\left(R_{\text {on-water }}\right)$.

\begin{tabular}{lrrr}
\hline \hline Systematic uncertainty & $R_{\text {water }}$ & \multicolumn{1}{c}{$R_{\text {air }}$} & $R_{\text {on-water }}$ \\
\hline MC statistics & 0.03 & 0.04 & 0.12 \\
PØD mass & 0.01 & 0.01 & 0.01 \\
PØD fiducial volume & $<0.01$ & $<0.01$ & $<0.01$ \\
PØD alignment & $<0.01$ & $<0.01$ & $<0.01$ \\
Energy scale & 0.05 & 0.05 & 0.10 \\
Hit matching & $<0.01$ & $<0.01$ & $<0.01$ \\
Track PID & 0.05 & 0.05 & 0.09 \\
Energy resolution & $<0.01$ & $<0.01$ & 0.01 \\
Angular resolution & $<0.01$ & $<0.01$ & 0.01 \\
Track median width & $<0.01$ & $<0.01$ & $<0.01$ \\
Shower median width & 0.04 & 0.04 & 0.08 \\
Shower charge fraction & 0.01 & 0.04 & 0.04 \\
Flux and cross sections & 0.07 & 0.09 & 0.06 \\
Total & 0.11 & 0.13 & 0.21 \\
\hline \hline
\end{tabular}

\section{Flux and cross section systematic uncertainties}

For the inclusion of the flux and cross section systematic uncertainties in the analysis, each analyzed MC event is reweighted according to the uncertainties of the flux and cross section parameters which are correlated. The parameter values and uncertainties are provided by different external measurements such as NA61 and other hadronic production experiments, and these parameters are then fitted to ND280 data from TPC and FGD, the other subdetectors of ND280 than PØD. The systematic parameters and their uncertainties obtained from the fit to the ND280 data, which includes 25 flux parameters, 6 FSI parameters, 2 NEUT parameters, and 13 neutrino interaction parameters, has been studied in Ref. [23].

To obtain the flux and cross section systematic uncertainties, the systematic parameters are thrown according to the covariance matrix and the analysis described in Sec. IV is then applied to each throw. The distributions are fit with single Gaussians and the resulting width is considered to be the flux and cross section systematic uncertainty for the analysis. The uncertainties obtained for water $\left(R_{\text {water }}\right)$, air $\left(R_{\text {air }}\right)$, and on-water $\left(R_{\text {on-water }}\right)$ are $0.07,0.09$, and 0.06 respectively.

\section{Summary of the systematic uncertainties}

All systematic uncertainties on the $\mathrm{CC} \nu_{e}$ data/MC ratios for water $\left(R_{\text {water }}\right)$, air $\left(R_{\text {air }}\right)$, and on-water $\left(R_{\text {on-water }}\right)$ that were estimated in the previous sections are summarized in Table IV. This table also shows the total systematic uncertainty.

\section{RESULTS}

The results obtained for the background subtracted data/ MC ratio $(R)$ for water configuration, air configuration, and on-water are

$$
\begin{aligned}
R_{\text {water }} & =0.89 \pm 0.08(\text { stat }) \pm 0.11(\text { sys }), \\
R_{\text {air }} & =0.90 \pm 0.09(\text { stat }) \pm 0.13(\text { sys }), \quad \text { and } \\
R_{\text {on-water }} & =0.87 \pm 0.33 \text { (stat) } \pm 0.21 \text { (sys) } .
\end{aligned}
$$

The ratios are consistent with 1 , within statistical and systematic uncertainties. For the on-water ratio, uncertainties are relatively large due to limited statistics and the impact of the subtraction method.

For the selected events, the distribution of the reconstructed particle directions is shown in Fig. 5 and the distribution of particle energies is shown in Fig. 6. This result indicates that the beam $\nu_{e}$ component in high energy region measured in the data is consistent with expectations after including constraints from the ND280 data for all configurations.
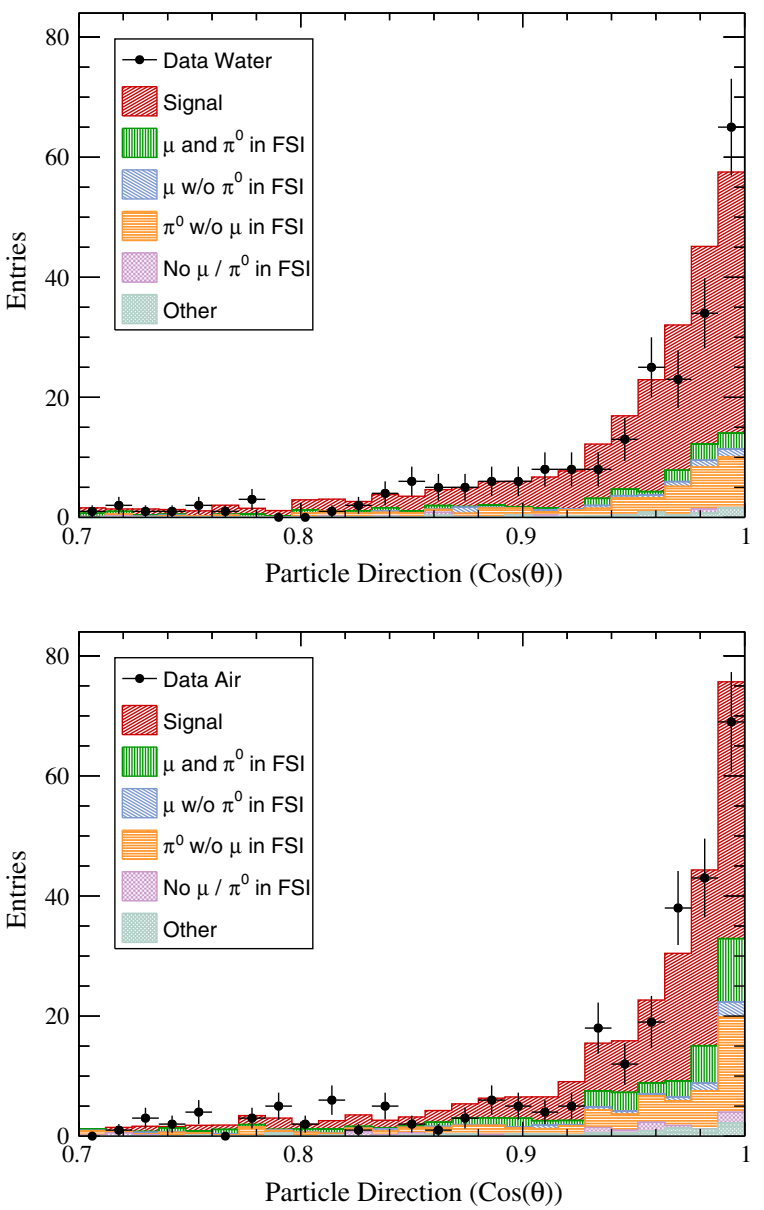

FIG. 5 (color online). Events passing the event selection as a function of the particle direction for water (top) and air configuration (bottom). The MC events are normalized to data POT, and the fit results from ND280 are applied. 

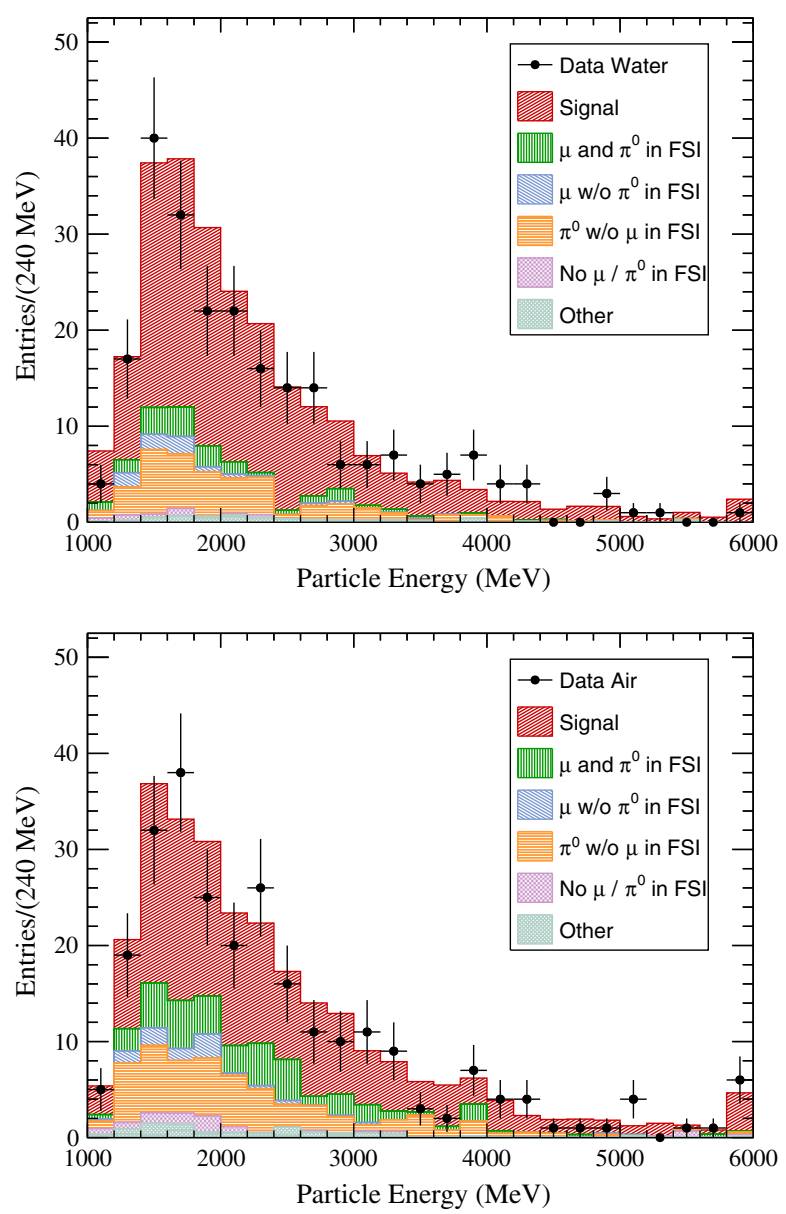

FIG. 6 (color online). Events passing the event selection as a function of the particle energy for water (top) and air configuration (bottom). The MC events are normalized to data POT, and the fit results from ND280 are applied.

\section{CONCLUSION}

In conclusion, measurements of $\mathrm{CC}_{e}$ interactions using the ND280 PØD have been made. The PØD includes fillable water targets which allows separate measurements for the water and air configurations of the ND280 PØD as well as the measurement of $\nu_{e}$ on-water interactions above $1.5 \mathrm{GeV}$ in a predominantly $\nu_{\mu}$ beam. About $\sim 85 \%$ of the selected sample comes from the decay of kaons.

The 230 (257) water configuration (air configuration) electron neutrino candidate events selected in the data are in good agreement with the prediction for the water configuration, the air configuration, and for the on-water subtraction samples respectively. The measurement is statistically limited, especially for on-water, but it will be improved in the future, since collection of 10 times more data is planned in the coming years. Furthermore, studies and improvements to the reconstruction algorithms are being investigated to lower the energy threshold, which will lead to the measurement of the $\nu_{e}$ cross section on water.

This is the first $\nu_{e}$ interaction rate measurement on water in the few $\mathrm{GeV}$ energy region. Interactions of $\nu_{e}$ on water are of particular interest for long-baseline neutrino oscillation experiments, and atmospheric neutrino experiments using water Cherenkov detectors with the aim to measure $C P$ violation in the lepton sector.

\section{ACKNOWLEDGMENTS}

We thank the J-PARC staff for superb accelerator performance and the CERN NA61 collaboration for providing valuable particle production data. We acknowledge the support of MEXT, Japan; NSERC, NRC and CFI, Canada; CEA and CNRS/IN2P3, France; DFG, Germany; INFN, Italy; National Science Centre (NCN), Poland; RSF, RFBR and MES, Russia; MINECO and ERDF funds, Spain; SNSF and SER, Switzerland; STFC, U.K.; and DOE, U.S.A. We also thank CERN for the UA1/NOMAD magnet, DESY for the HERA-B magnet mover system, NII for SINET4, the WestGrid and SciNet consortia in Compute Canada, GridPP, U.K. In addition participation of individual researchers and institutions has been further supported by funds from: ERC (FP7), EU; JSPS, Japan; Royal Society, U.K.; DOE Early Career program, U.S.A.
[1] Y. Hayato, Nucl. Phys. B, Proc. Suppl. 112, 171 (2002).

[2] J. Blietschau et al. (Gargamelle Collaboration), Nucl. Phys. B133, 205 (1978).

[3] L. B. Auerbach et al. (LSND Collaboration), Phys. Rev. D 63, 112001 (2001).

[4] J. A. Formaggio and G. P. Zeller, Rev. Mod. Phys. 84, 1307 (2012).

[5] K. Abe et al. (T2K Collaboration), Nucl. Instrum. Methods Phys. Res., Sect. A 659, 106 (2011).

[6] J. Arafune, M. Koike, and J. Sato, Phys. Rev. D 56, 3093 (1997).
[7] K. Abe et al. (T2K Collaboration), Phys. Rev. Lett. 112, 061802 (2014).

[8] K. Abe et al. (T2K Collaboration), Phys. Rev. D 87, 012001 (2013).

[9] K. Abe et al., arXiv:1109.3262.

[10] C. Adams et al. (LBNE Collaboration), arXiv:1307.7335.

[11] A. Stahl et al., Report No. CERN-PH-EP-2012-021, SPSCEOI-007, 2012.

[12] K. Abe et al. (T2K Collaboration), Phys. Rev. D 89, 099902 (2014). 
[13] S. Assylbekov et al. (T2K ND280 P0D Collaboration), Nucl. Instrum. Methods Phys. Res., Sect. A 686, 48 (2012).

[14] B. G. Tice et al. (Minerva Collaboration), Phys. Rev. Lett. 112, 231801 (2014).

[15] B. Tice, Ph.D. thesis, Rutgers University, 2014.

[16] M. Day and K. S. McFarland, Phys. Rev. D 86, 053003 (2012).

[17] K.A. Olive et al. (Particle Data Group), Chin. Phys. C 38, 090001 (2014).

[18] G. Gran et al., Phys. Rev. D 74, 052002 (2006).

[19] C. Mariani et al., Phys. Rev. D 83, 054023 (2011).

[20] A. Rodriguez et al., Phys. Rev. D 78, 032003 (2008).

[21] S. Nakayama et al., Phys. Rev. Lett. 619, 255 (2005).

[22] M. Hasegawa et al., Phys. Rev. Lett. 95, 252301 (2005).
[23] K. Abe et al. (T2K Collaboration), Phys. Rev. D 89, 092003 (2014).

[24] A. Schalicke, A. Bagulya, Ø. Dale, F. Dupertuis, V. Ivanchenko, O. Kadri, A. Lechner, M. Maire, M. Tsagri, and L. Urban, J. Phys. Conf. Ser. 331, 032029 (2011).

[25] O. Kadri, V. N. Ivanchenko, F. Gharbi, and A. Trabelsi, Nucl. Instrum. Methods Phys. Res., Sect. B 258, 381 (2007).

[26] D. L. Sawkey and B. A. Faddegon, Med. Phys. 36, 698 (2009).

[27] V. N. Ivanchenko, Nucl. Instrum. Methods Phys. Res., Sect. A 502, 666 (2003).

[28] A. N. Kolmogorov, G. Ist. Ital. Attuari 4, 83 (1933).

[29] N. Smirnov, Ann. Math. Stat. 19, 279 (1948). 